\title{
OCTOBRE 1973
}

55• ANNÉE - N• 658
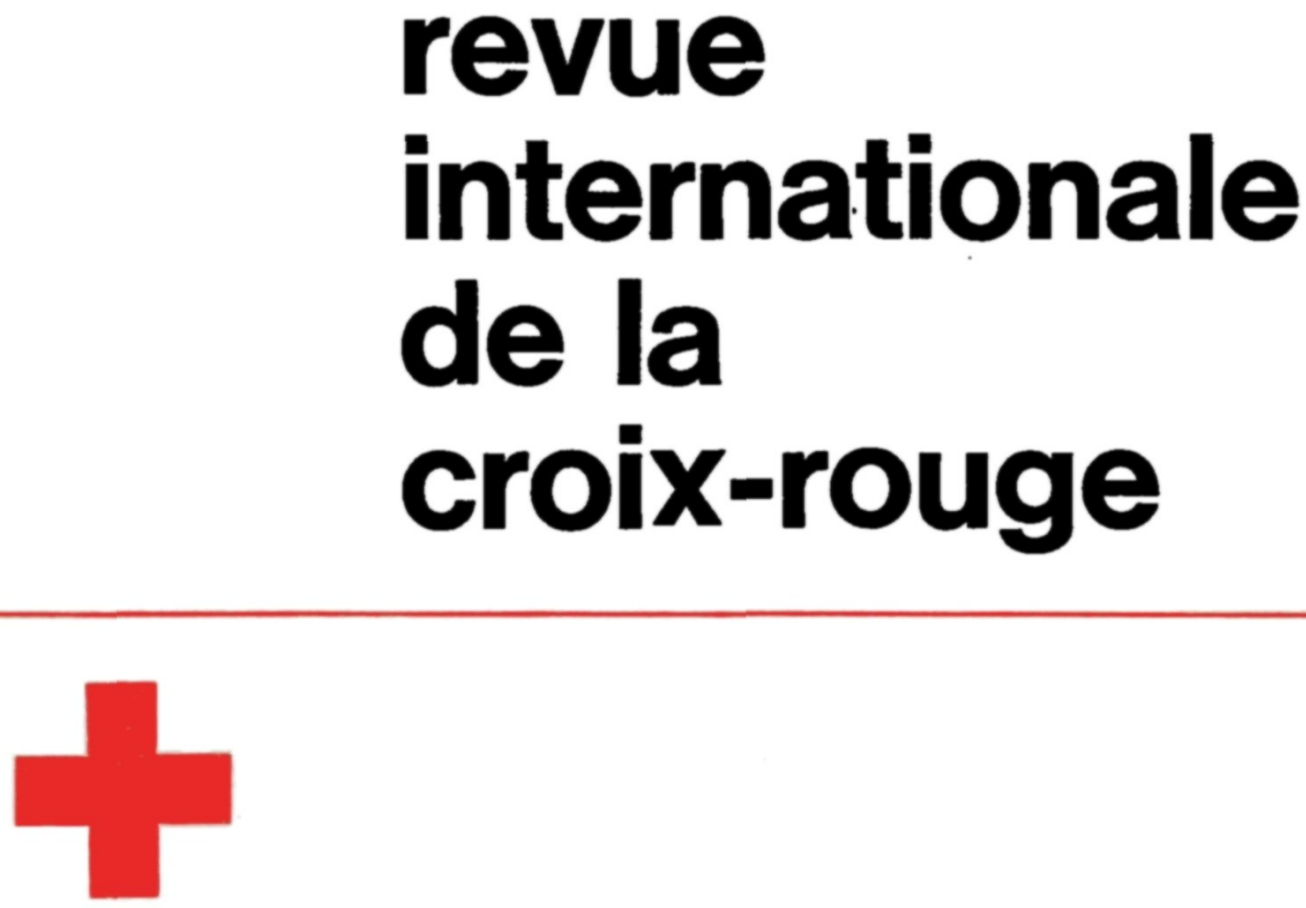

INTER ARMA CARITAS

GENÈVE

COMITÉ INTERNATIONAL DE LA CROIX-ROUGE FONDÉ EN 1863 


\title{
COMITÉ INTERNATIONAL DE LA CROIX-ROUGE
}

MM. ERIC MARTIN, docteur en médecine, professeur honoraire de l'Université, Genève, président (membre depuis 1973)

JEAN PICTET, docteur en droit, président de la Commission juridique, vice-président (1967)

HARALD HUBER, docteur en droit, juge fédéral, vice-président (1969)

HANS BACHMANN, docteur en droit, directeur des finances de la ville, Winterthour (1958)

DIETRICH SCHINDLER, docteur en droit, professeur à l'Université, Zurich (1961)

M"le MARJORIE DUVILLARD, infirnière, ancienne directrice de l'Ecole d'infirmières du Bon Secours, Genève (1961)

MM. MAX PETITPIERRE, docteur en droit, ancien conseiller fédéral (1961)

ADOLPHE GRAEDEL, ancien député au Conseil national, ancien secrétaire général de la Fédé. ration internationale des ouvriers sur métaux (1965)

$M^{m e}$ DENISE BINDSCHEDLER-ROBERT, docteur en droit, professeur à l'Institut universitaire de hautes études internationales, Genève (1967)

MM. MARCEL A. NAVILLE, licencié ès lettres, ancien directeur de banque, président du CICR de 1969 à 1973 (1967)

JACQUES F. DE ROUGEMONT, docteur en médecine (1967)

ROGER GALLOPIN, docteur en droit, ancien directeur général du CICR (1967)

WALDEMAR JUCKER, docteur en droit, secrétaire de l'Union syndicale suisse (1967)

VICTOR H. UMBRICHT, docteur en droit, administrateur (1970)

PIERRE MICHELI, licencié en droit, ancien ambassadeur (1971)

PIERRE BOISSIER, licencié en droit, directeur de 1'Institut Henry-Dunant, Genève (1973)

GILBERT ETIENNE, docteur en droit, professeur a l'Institut universitaire de hautes études internationales, Gendve (1973)

ULRICH MIDDENDORP, docteur en médecine, chef de la clinique chirurgicale de l'Hôpital cantonal, Winterthour (1973)

M'l MARION ROTHENBACH, chargée de cours à l'Ecole des sciences sociales et politiques de l'Université, Lausanne (1973)

Membres honoraires: M. JACQUES CHENEVIËRE, vice-président d'honneur; M ${ }^{\text {He }}$ LUCIE ODIER, vice-présidente d'honneur; MM. GUILLAUME BORDIER, CARL J. BURCKHARDT, PAUL CARRY, M ${ }^{m}$ MARGUERITE GAUTIER-VAN BERCHEM, MM. SAMUEL A. GONARD, EDOUARD DE HALLER, RODOLFO OLGIATI, PAUL RUEGGER, FRÉDÉRIC SIORDET, ALFREDO VANNOTTI, ADOLF VISCHER.

\section{CONSEIL EXÉCUTIF}

\author{
M. ROGER GALLOPIN, président \\ M. VICTOR H. UMBRICHT, vice-président \\ $M^{\text {me }}$ DENISE BINDSCHEDLER-ROBERT \\ D ULRICH MIDDENDORP \\ M. GOTTFRIED DE SMIT
}




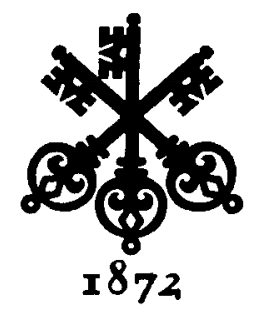

PRÉSENTE SUR LES CINQ CONTINENTS, LA GRANDE BANQUE AUX TROIS CLEFS EST A VOTRE DISPOSITION POUR EFFECTUER TOUTES VOS OPÉRATIONS FINANCIĖRES

\section{SOCIÉTÉ DE BANQUE SUISSE}

SCHWEIZERISCHER BANKVEREIN - SOCIETÁ DI BANCA SVIZZERA

SWISS BANK CORPORATION

SIËGE DE GENËVE

2, RUE DE LA CONFÉDÉRATION

TÉL. 201111

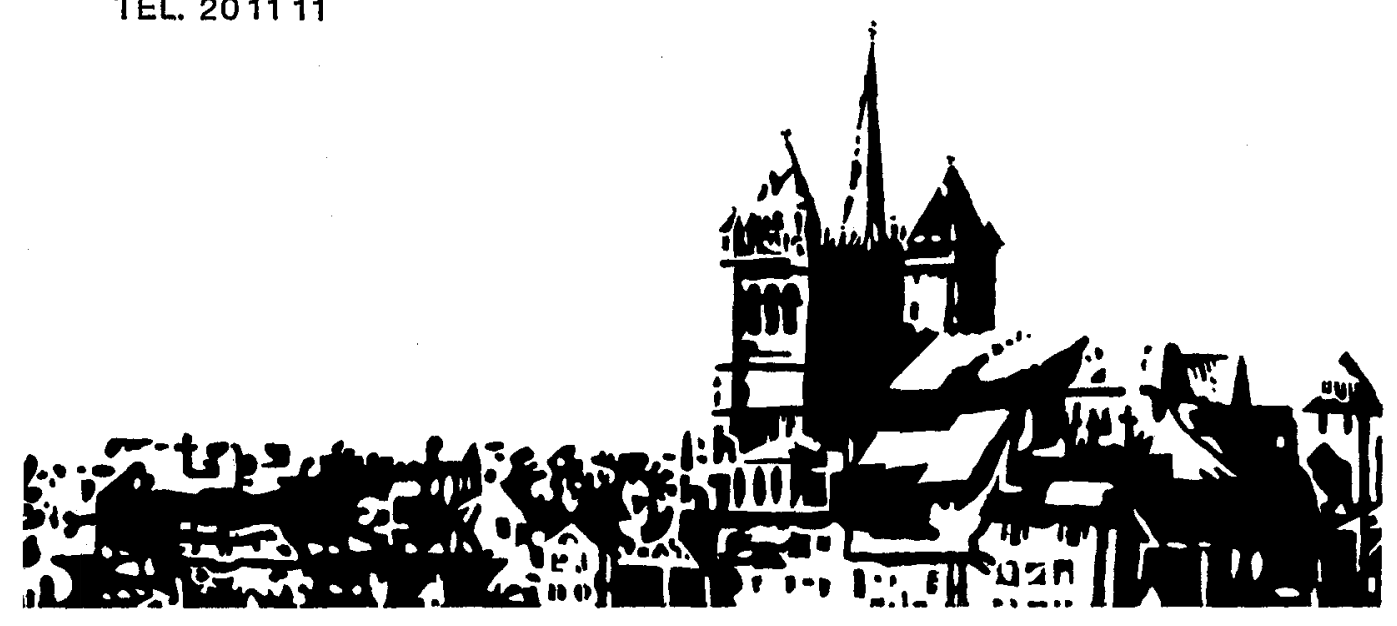




\section{SOCIETE FIDUCIAIRE ROMANIDE OFOR S.A.}

Place Saint-Gervais 1, Genève - Tél. 317050

- Expertises, revisions et organisations comptables

^ Domiciliation et administration de sociétés

Assainissements et liquidations

Interventions et conseils en matière fiscale

\section{JOURIAL DR GEIÈVE}

deux imprimeries

deux adresses

rue François-Dussaud 20

1211 Genève 8 - Tóléphone 431940

rue Général-Dufour 5-7

1211 Genève 11 - Téléphone 250350

TYPO - OFFSET - ROTATIVE 


\section{SOMMAIRE}

COMITE INTERNATIONAL DE LA CROIX-ROUGE

\section{DANS LE MONDE DE LA CROIX-ROUGE}

\section{FAITS ET DOCUMENTS}

\section{LIVRES ET REVUES}

\section{REVUE INTERNATIONALE \\ DE LA CROIX-ROUGE}

OCTOBRE 1973 - № 658

Projets de protocoles additionnels aux Conventions de Genève - Analyse succincte . . . . . . . . 581

Convocation de la Conférence diplomatique . . . 590

Reconnaissance de la Société de la Croix-Rouge de Fidji (490' circulaire aux Comités centraux).

Reconnaissance de la Société de la Croix-Rouge du Bangladesh (491e circulaire aux Comités centraux) .. . . . . . . . . . . . . . .

Reconnaissance de la Société de la Croix-Rouge de Singapour $\left(492^{e}\right.$ circulaire aux Comités cen$\operatorname{traux})$..............

Activités extérieures:

Burundi - Chili - Sous-continent asiatique Moyen-Orient - République arabe du Yémen

Comment des familles sont réunies grâce à la Croix-Rouge ........... 605

Institut régional de formation pour l'Afrique centrale . . . . . . . . . . . . . . . 608

Rapport annuel de la Ligue . . . . . . . . . 609 Assistance internationale de la Croix-Rouge en Indochine . . . . . . . . . . . . . . 611

Birmanie . . . . . . . . . . . . 615

Diffusion des Conventions de Genève: Japon Tanzanie . . . . . . . . . . . . 616

Colloque à l'Institut international de droit humanitaire . . . . . . . . . . . . . . . 619 Congrès d'Amnesty International . . . . . . 623 Un vote en faveur de la Croix-Rouge . . . . . 623 Une nouvelle image professionnelle de l'infirmière 625 La protection internationale des réfugiés . . . 627 


\section{INTERNATIONAL \\ REVIEW \\ OF THE RED CROSS}

\section{SUPPLEMENTS \\ De la ReVue}

EN LANGUE ESPAGNOLE

EN LANGUE ALLEMANDE

\section{REVUE INTERNATIONALE DE LA CROIX-ROUGE}

Une édition en langue anglaise paraît chaque mois. Elle est en principe identique à l'édition française, et peut être obtenue aux mêmes conditions.
Proyectos de Protocolos adicionales a los Convenios de Ginebra - Breve análisis - Reconocimiento de la Sociedad de la Cruz Roja de Fidji - Reconocimiento de la Sociedad de la Cruz Roja de Bangla Desh Reconocimiento de la Sociedad de la Cruz Roja de Singapur (Circulares núm. 490-491-492) - En beneficio de las víctimas de Chile - Una votación a favor de la Cruz Roja.

Entwurf von Zusatzprotokollen zu den Genfer Abkommen - Zusammengefasste Analyse - Drei neue Nationale Rotkreuzgesellschaften - Für die Opfer in Chile - Tätigkeiten des Henry-Dunant-Instituts.

Elle est publiée chaque mois par le Comité international de la Croix-Rouge.

7, avenue de la Paix, 1211 Genève 1 (Suisse) - Compte de chèques postaux $12-1767$.

Abonnement un an: Fr. 30,-; le numéro: Fr. 3,-.

RÉDACTION : J.-G. LOSSIER

Seuls les textes signés par le Comité international de la Croix-Rouge engagent la responsabilité de celui-ci. 